EPJ Web of Conferences 59, 04005 (2013)

DOI: $10.1051 /$ epjconf/20135904005

(C) Owned by the authors, published by EDP Sciences, 2013

\title{
Defect-induced mix experiment for NIF
}

\author{
M.J. Schmitt', P.A. Bradley ${ }^{1}$, J.A. Cobble ${ }^{1}$, S.C. Hsu ${ }^{1}$, N.S. Krasheninnikova ${ }^{1}$, \\ G.A. Kyrala ${ }^{1}$, G.R. Magelssen ${ }^{1}$, T.J. Murphy ${ }^{1}$, K.A. Obrey ${ }^{1}$, I.L. Tregillis ${ }^{1}$, \\ F.J. Wysocki ${ }^{1}$ and S.M. Finnegan ${ }^{2}$
}

${ }^{1}$ Los Alamos National Laboratory, MS F699, Los Alamos, NM 87544, USA

2 Office of Fusion Energy Sciences, SC-24, 19901 Germantown Rd, Germantown, MD 20874, USA

\begin{abstract}
The Defect Induced Mix Experiment (DIME-II) will measure the implosion and mix characteristics of $\mathrm{CH}$ capsules filled with 5 atmospheres of DT by incorporating mid-Z dopant layers of Ge and Ga. This polar direct drive (PDD) experiment also will demonstrate the filling of a CH capsule at target chamber center using a fill tube. Diagnostics for these experiments include areal x-ray backlighting to obtain early time images of the implosion trajectory and a multiple-monochromatic imager (MMI) to collect spectrally-resolved images of the capsule dopant line emission near bangtime. The inclusion of two (or more) thin dopant layers at separate depths within the capsule shell facilitates spatial correlation of mix between the layers and the hot gas core on a single shot. The dopant layers are typically $2 \mu \mathrm{m}$ thick and contain dopant concentrations of $1.5 \%$. Three dimensional Hydra simulations have been performed to assess the effects of PDD asymmetry on capsule performance.
\end{abstract}

\section{INTRODUCTION}

Quantifying $4 \pi$ mix and defect-induced mix in ICF implosions is needed to achieve robust ignition and to set tolerances on heterogeneities in capsule construction and irradiation for future Inertial Fusion Energy (IFE) facilities. For the past two years, LANL has conducted feature-driven mix implosion experiments at the Laboratory for Laser Energetics (LLE) at the University of Rochester using the Omega Laser Facility [1]. These experiments have demonstrated the technique of using capsules with pre-engineered equatorial grooves combined with thin mid-Z doped layers placed strategically within the capsules to interrogate mix near the gas-shell interface. Using spectral imaging techniques pioneered by Mancini and his team at the University of Nevada-Reno [2], 2-D spectrally-resolved images capable of determining where doped material mixes into the hot gas can be generated. The mix layer width can be determined to the micron level (even using $\mathrm{x}$-ray imaging with $\sim 15 \mu \mathrm{m}$ resolution) by varying the distance of the doped layer from the gas/shell interface. The addition of pre-engineered features into these capsules provides additional information on how high mode number perturbations enhance the normal $4 \pi$ mix.

In FY12 we plan on using our Omega-developed experimental techniques on NIF where the impact of mix and high mode number features can be imaged using both spectral and nuclear diagnostics. Dubbed the Defect Induced Mix Experiment (DIME-II), this campaign will measure the mix characteristics of $2.2 \mathrm{~mm}$ diameter, $42 \mu \mathrm{m}$ thick $\mathrm{CH}$ capsules imploded using NIF's polar direct drive (PDD) laser illumination geometry. The implosion trajectory of the capsule will be fully imaged in 2-D using X-ray backlighting. Moreover, a LANL-built multiple monochromatic imager (MMI) is being built to image x-ray line emission from mid-Z dopants including $\mathrm{Ge}, \mathrm{Ga}$ and $\mathrm{Cu}$ in the $8-13 \mathrm{keV}$

This is an Open Access article distributed under the terms of the Creative Commons Attribution License 2.0, which permits unrestricted use, distribution, and reproduction in any medium, provided the original work is properly cited. 


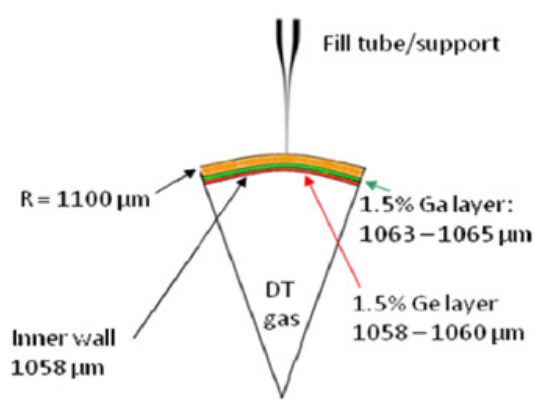

(a)

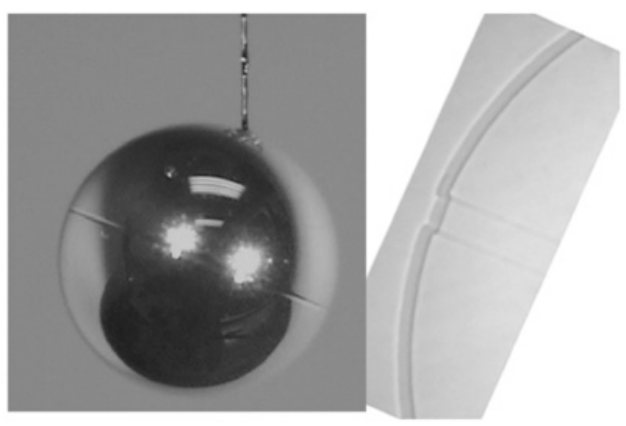

(b)

Figure 1. (a) NIF CH capsule showing fill tube and layers containing Ge and Ga dopants and (b) a view of a capsule with an equatorial feature along with a blow up of the feature cross-section.

spectral range near the end of the implosion when $P d V$ work on the gas heats the mix layer above $1 \mathrm{keV}$ in temperature. These capsules will be filled with 5 atmospheres of DT gas to provide nuclear yield in the $10^{14}$ neutron regime. Imaging of the burn in these capsules will be provided by NIF's neutron imager (NI) for cross-correlation with spectral images from the doped layers.

\section{MID-Z DOPED LAYER EXPERIMENTS FOR FY12}

Thin shell CH capsules were chosen for our NIF experiments since they can be precisely doped with mid-Z ions and their inner surface can easily be modified by machining of the mandrel to incorporate pre-engineered high mode number features. The capsules for our FY12 NIF shots will incorporate a $30 \mu \mathrm{m}$ OD fill tube (for DT filling at target chamber center) and two doped layers as shown in Fig. 1(a). The first DIME shots on NIF will incorporate a $2 \mu \mathrm{m}$ thick, $1.5 \%$ by atom Ge-doped $\mathrm{CH}$ layer on the inside surface of the capsule. This capsule will provide baseline mix information on the spectral flux from the dopant and its uniformity around the capsule. This will be followed by a capsule with the same dopant layer, but with the addition of a $80 \mu \mathrm{m}$ wide by $10 \mu \mathrm{m}$ deep equatorial groove, as depicted in Fig. 1(b). The results from this capsule will provide data on additional mix induced by the presence of the feature. Follow-on shots will repeat these experiments with two doped layers to confirm the mix layer width and measure enhanced mix in the defect region.

The configuration for these NIF shots is shown in Fig. 2(a). The capsules will be positioned using either the CryoTARPOS or the TARPOS. Both target positioning configurations are being carried along to allow flexibility for fielding these shots. The two equatorial DIMs will be used; one to hold the neutron imaging pinhole (located at polar-azimuthal angles, 90-315) and the other to hold an hGXI framing camera (90-78) needed to record the X-ray backlit images. Details of the backlighter are shown in Fig. 2(b) where a $2 \mathrm{~mm}$ diameter foil is held $5 \mathrm{~mm}$ from TCC by a stalk off the capsule fill tube mount. The foil will be heated by two of NIF's 48 quads as shown in Fig. 2(b). MMI X-ray spectral images will be collected from the polar diagnostic insertion module (DIM). These three imaging diagnostics will provide the maximum spatial information for the measurement of $4 \pi$ mix and additional 3 -D mix induced by the presence of the feature.

\section{PDD SYMMETRY AND BACKLIGHTER DESIGN}

Polar direct drive of capsules on NIF requires the repointing and defocusing of the laser cones to minimize pole-to-equator variations in the laser drive illuminating the capsules. The pointing configuration for the NIF DIME shots is shown in Fig. 3. The geometric center of each cone strikes 


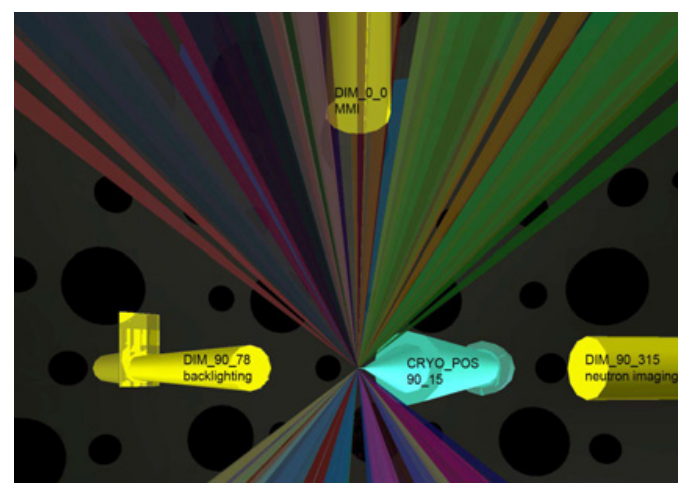

(a)

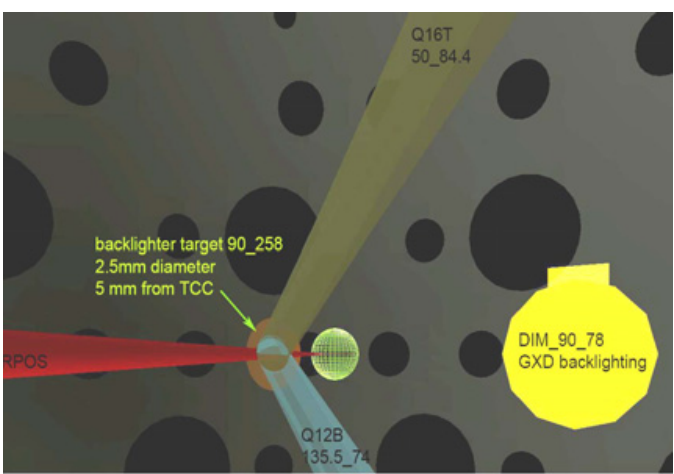

(b)

Figure 2. (a) NIF DIME configuration showing the PDD laser beams converging on the capsule being held in this rendition by the cryoTARPOS along with the imaging diagnostics shown in yellow. The backlighter foil mounting is shown in (b) where it is illuminated by two laser quads; Q50_84.4 from above and Q135.5_74 from below. Both quads strike the foil on the front side facing the capsule.

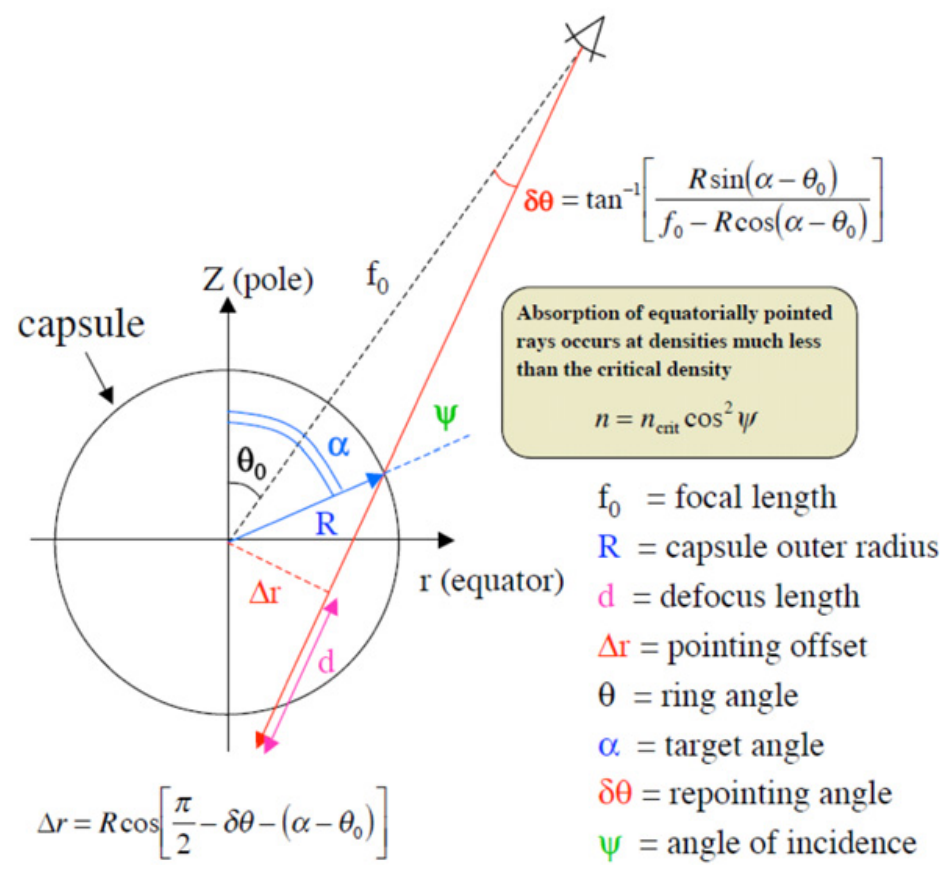

Figure 3. Schematic and mathematical relation between the angles and distances needed to repoint the beams for PDD.

the capsule at polar angle, $\alpha$, and is defocused a distance, $d$, beyond its normal TCC focal point. The pointing and defocusing parameters are given in Table 1. These pointing specifications were provided by Steve Craxton of LLE at the U. of Rochester for a hybrid CH-over-glass capsule of similar mass and diameter to our pure $\mathrm{CH}$ capsule. Note that the quads of the $44.5^{\circ}$ cone have been split (into two upper "a" beams and two lower "b" beams) to further smooth the polar illumination profile. Final adjustment of these pointing parameters is currently being performed. 
Table 1. Polar pointing parameters for the NIF 700kJ DIME shots.

\begin{tabular}{|l|c|c|c|c|}
\hline Ring: $\theta_{0}$ & Target: $\alpha$ & Defocus $(\mathrm{cm})$ & xspot $\Delta \mathrm{x}(\mu \mathrm{m})$ & yspot $\Delta \mathrm{x}(\mu \mathrm{m})$ \\
\hline 23.5 & 31.56 & 1.8 & 631 & 882 \\
30.0 & 38.06 & 1.9 & 590 & 824 \\
$45.5 \mathrm{a}$ & 42.19 & 2.4 & 367 & 635 \\
$44.5 \mathrm{~b}$ & 80.99 & 1.6 & 367 & 635 \\
50.0 & 84.10 & 1.2 & 343 & 593 \\
\hline
\end{tabular}

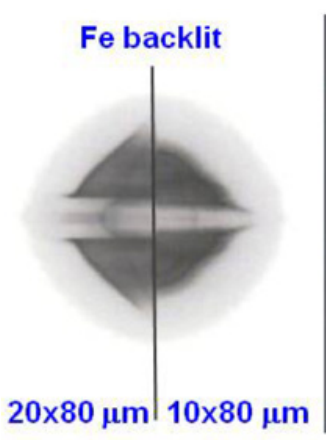

(a)

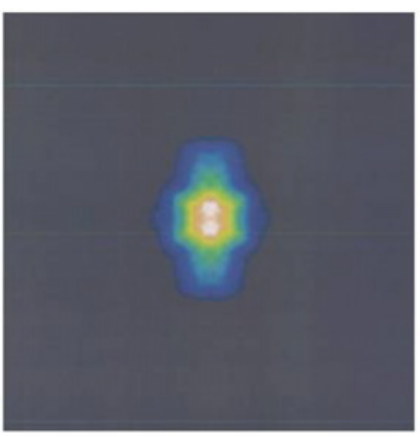

(b)

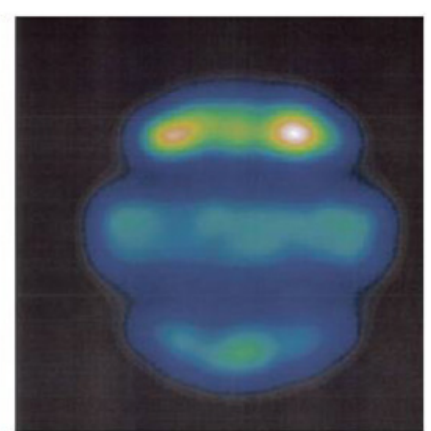

(c)

Figure 4. Simulated DIME (a) backlit radiograph for two groove sizes showing gap from defect (500 $\mu$ m outer halo edge radius) prior to core self-emission, (b) neutron image and (c) MMI image for the CH capsule with an equatorial defect at bangtime (spatial and spectral variations in the vertical direction). All images show clear effects from the feature. When the defect is absent, the central gap in the radiograph and the central emission band in the MMI image do not occur and the neutron image is round. Black image mats are $200 \mu \mathrm{m}$ squares.

As previously mentioned, $\mathrm{x}$-ray backlighting requires the removal of two NIF quads from the capsule drive. This has been simulated in 3-D [3] using the rad-hydro code Hydra [4]. Uncompensated removal of the two quads produces a bulge in the capsule implosion and a $70 \%$ reduction in yield.

Increasing the energy of the remaining seven quads in the affected beam cones by $8 / 7$ ths and symmetrically adjusting their positions around the capsule circumference (to compensate for each of the missing backlighter quads) effectively eliminates any reduction in yield and symmetry. Additional calculations have been performed to simulate both the backlit radiograph and the MMI images obtained using the hGXI cameras. Simulated diagnostic images are given in Fig. 4.

\section{CONCLUSIONS}

The design of a NIF experiment to measure both $4 \pi$ and feature-induced mix in ICF implosions has been performed. Based on demonstration experiments performed at Omega over the past two years, the DIME experiments will provide both spectral and neutron images needed to build a predictive capability for obtaining robust ICF ignition and designing future IFE facilities.

\section{References}

[1] P.A. Bradley, J.A. Cobble, S.C. Hsu, G.R. Magelssen, T.J. Murphy, M.J. Schmitt, I.L. Tregillis, N.S. Vinyard, F.J. Wysocki, and S.M. Finnegan, these proceedings 


\section{IFSA 2011}

[2] T. Nagayama, R. Mancini, R. Florido, R. Tommasini, J. Koch, J. Delettrez, S. Regan, V. Smalyuk, L. Welser, and I. Golovkin, Rev. Sci. Instrum. 79, 10E921 (2008)

[3] N. S. Krasheninnikov, M. J. Schmitt and S. M. Finnegan, Phys Plasmas 19, 012702 (2012)

[4] M. M. Marinak, R. E. Tipton, O. L. Landen, T. J. Murphy, P. Amendt, S. W. Haan, S. P. Hatchett, C. J. Keane, R. McEachern, and R. Wallace, Phys. Plasmas 3, 2070 (1996) 